\title{
Nível de flexibilidade obtida pelo teste de sentar e alcançar a partir de estudo realizado na Grande São Paulo
}

\author{
Level of flexibility through sit and reach test from research \\ performed in São Paulo city
}

\author{
Cibele Calvi Anic Ribeiro ${ }^{1}$ \\ Cesar Cavinato Cal Abad \\ Ronaldo Vilela Barros \\ Turíbio Leite de Barros Neto ${ }^{1}$
}

1. Universidade Federal de São Paulo, Centro de Medicina da Atividade Física e do Esporte. São Paulo, SP. Brasil.

2. Universidade Bandeirante de São Paulo, Instituto de Educação Desportiva, São Paulo, SP, Brasil.

3. Universidade de São Paulo, Laboratório de Desempenho Esportivo São Paulo, SP. Brasil.

Recebido em 11/12/09 Revidado em 30/05/10 Aprovado em 06/07/10
Resumo - O objetivo do estudo foi identificar o nível de flexibilidade de homens e mulheres de diferentes faixas etárias através do teste de sentar e alcançar seguindo protocolo do Canadian Standardized Test of Fitness (CSTF); classificá-los de acordo com a tabela sugerida pelo mesmo e, a partir dos resultados obtidos elaborar uma nova tabela normativa que reflita a população estudada. Participaram do estudo 16.405 sujeitos (11.114 mulheres e 5.291 homens) praticantes e não praticantes de exercícios físicos regulares, divididos em grupos etários: 15 a 19 ( $\mathrm{n=954),} 20$ a 29 ( $\mathrm{n}=2916), 30$ a 39 ( $\mathrm{n}=2161), 40$ a 49 (n=2333), 50 a $59(\mathrm{n}=2739), 60$ a $69(\mathrm{n}=3195)$, acima de $70(\mathrm{n}=2107)$. Os percentis foram calculados com escores do teste original e como pontos de corte considerou-se os percentis 20, 40, 60 e 80 para classificação fraco, abaixo da média, média, acima da média, excelente, respectivamente. De acordo com as classificações do CSTF as faixas etárias entre 15 e 39 anos foram classificadas como fraco, com média de flexibilidade variando entre $24,80 \pm 9,68 \mathrm{~cm}$ e $26,13 \pm 10,11 \mathrm{~cm}$ no feminino e entre $21,48 \pm 9,90 \mathrm{~cm}$ e $22,84 \pm 9,64 \mathrm{~cm}$ no masculino. Nas categorias de $40 \mathrm{a}$ 69 a média variou entre $22,76 \pm 9,62 \mathrm{~cm}$ e $25,39 \pm 9,54 \mathrm{~cm}$ no feminino e entre $16,39 \pm 10,13 \mathrm{~cm}$ e $19,93 \pm 9,19 \mathrm{~cm}$ no masculino, ficando abaixo da média. Apesar de a maioria da amostra ser praticante de exercício físico regular, a média do nível de flexibilidade apresentada não correspondeu à média sugerida pela tabela canadense demonstrando a importância de se construir tabelas de referências nacionais e se estabelecer novos valores normativos como a tabela proposta pelo presente trabalho.

Palavras-chave: Amplitude de movimento articular; Maleabilidade; Aptidão física; Saúde.

Abstract - The objective of this study was to examine the level of flexibility in men and women of different ages by the sit-and-reach test and to classify them according to the Canadian Standardized Test of Fitness (CSTF). The results were used to elaborate a new table that reflects the population studied. The sample consisted of 16,405 physically active and inactive subjects who were divided according to age: 15 to $19(n=954), 20$ to $29(n=2916), 30$ to $39(n=2161), 40$ to $49(n=2333), 50$ to $59(n=2739), 60$ to $69(n=3195)$, and $>70$ years $(n=2107)$. Percentiles were calculated using the original test scores and the $20^{\text {th }}, 40^{\text {th }}, 60^{\text {th }}$ and $80^{\text {th }}$ percentiles were used as cut-offs for classification as poor, below the average, average, above the average and excellent, respectively. According to the CSTF classification, the age groups from 15 to 39 years were classified as poor, with mean flexibility ranging from $24.805 \pm 9.684$ to $26.130 \pm 10.111 \mathrm{~cm}$ in women and from $21.480 \pm 9.905$ to $22.848 \pm 9.648 \mathrm{~cm}$ in men. In the 40- to 69-year age groups, mean flexibility ranged from $22.768 \pm 9.627$ to $25.396 \pm 9.547$ in women and from $16.396 \pm 10.136$ to $19.935 \pm 9.192 \mathrm{~cm}$ in men and was classified as below the average. Although most of the subjects performed regular exercise, the mean flexibility level observed did not correspond to the average suggested by the CSTF, demonstrating the importance of elaborating national reference tables and of establishing new normative values such as the table proposed in this study.

Key words: Range of motion; Malleability; Physical fitness; Health. 


\section{INTRODUÇÃO}

Definida como a capacidade de uma articulação se mover em uma amplitude ótima de movimento ${ }^{1}$ a flexibilidade ganhou especial atenção nos últimos 20 anos principalmente depois que instituições importantes como o American College of Sports Medicine (ACSM) e o American Heart Association passaram a recomendar seu treinamento para diferentes populações 2,3 $^{2}$.

As diretrizes são para que o treinamento de flexibilidade seja feito para os principais grupos musculares, com freqüência mínima de 3 vezes por semana, de 3 a 5 repetições cada grupo muscular e duração de 10 a 60 s cada repetição ${ }^{4}$. Os exercícios para flexibilidade podem ser realizados de forma ativa, passiva, estática, dinâmica, balística, com ou sem facilitação proprioceptiva sendo o nível de condicionamento do praticante e o objetivo do treino que determinarão que tipo de exercício ele deverá realizar ${ }^{5}$.

Antes do início de um programa de treinamento a realização de uma boa avaliação da flexibilidade se torna imprescindível. Um dos testes mais utilizados para este fim é o teste de sentar e alcançar (TSA) proposto por Wells e Dillon ${ }^{6}$ em 1952 que devido sua fácil aplicação e baixo custo operacional é recomendado e utilizado pelas principais baterias de testes já padronizadas em todo o mundo ( $\mathrm{Ca}$ nadian Standardized Test of Fitness; YMCA Physical Test Battery; FITNESSGRAM; Eurofit; President's Challenge Fitness Test; Celafiscs).

Os resultados do TSA permitem avaliações baseadas em tabelas normativas pré-elaboradas conforme gênero e idade ${ }^{7}$, porém, em sua grande maioria, elas se baseiam em populações canadenses ou americanas, havendo na literatura carência de dados que permitam a construção de tabelas normativas para outras populações, inclusive a brasileira, já que a flexibilidade é determinada, entre outros fatores como gênero e idade, por etnia e genética ${ }^{8}$.

No Brasil pelo fato de haver grande miscigenação populacional, melhor do que a construção de uma tabela normativa generalizada seria a elaboração de várias tabelas regionais que permitissem comparações intra e entre regiões, contudo, até onde sabemos há escassez de estudos que tenham se proposto a esta finalidade.

O presente trabalho foi realizado com dois objetivos principais: 1) identificar a flexibilidade de homens e mulheres, de diferentes faixas etárias e níveis de condicionamento, freqüentadores de unidades de uma rede de complexos esportivos no Estado de São Paulo e 2) Elaborar uma tabela normativa que sirva de referencial para avaliação e prescrição de exercício para melhora da flexibilidade desta população.

\section{PROCEDIMENTOS METODOLÓGICOS}

Antes do estudo, os indivíduos responderam anamnese. Os sujeitos foram esclarecidos dos procedimentos e aqueles que autorizaram a utilização de seus dados foram incluidos na amostra. Todos assinaram o de termo de consentimento livre e esclarecido.

Para participar do estudo os indivíduos deveriam ter ausência de história de doença ortopédica, reumatológica ou neurológica relacionadas com os membros inferiores, pelve ou coluna lombar.

O presente trabalho seguiu as recomendações da Resolução 196/96 do Conselho Nacional de Saúde para estudos com seres humanos e foi aprovado pelo comitê de ética da Universidade Federal de São Paulo - Escola Paulista de Medicina $(1695 / 05)$.

\section{Participantes}

Participaram do estudo 11.114 mulheres e 5.291 homens (16.405 no total) com idade entre 15 e 99 anos, freqüentadores de uma rede de complexos esportivos de unidades localizadas em diferentes regiões do Estado de São Paulo (Capital, Santo André, São Caetano, Campinas e Santos).

Conforme gênero os grupos foram divididos por faixa etária da seguinte forma: 15 a 19 anos ( $\mathrm{n}=954)$; 20 a 29 anos $(\mathrm{n}=2916) ; 30$ a 39 anos $(\mathrm{n}=2161) ; 40$ a 49 anos (n=2333); 50 a 59 anos (n=2739); 60 a 69 anos $(\mathrm{n}=3195)$ e acima de 70 anos $(\mathrm{n}=2107)$. Em cada faixa etária houve uma subdivisão dos indivíduos em ativos e sedentários de acordo com o nível de atividade física para que não se caracterizasse uma amostra tendenciosa.

Teste

O teste utilizado para avaliar a flexibilidade dos músculos isquiotibiais foi o de Sentar e Alcançar proposto originalmente por Wells e Dillon ${ }^{6}$ em 1952, seguindo a padronização canadense para os testes de avaliação da aptidão física do Canadian Standardized Test of Fitness (CSTF) 9 .

$\mathrm{O}$ teste é realizado numa caixa medindo 30,5 $\mathrm{cm} \times 30,5 \mathrm{~cm} \times 30,5 \mathrm{~cm}$ com uma escala de $26,0 \mathrm{~cm}$ em seu prolongamento, sendo que o ponto zero se encontra na extremidade mais próxima do avaliado 
e o $26^{\circ} \mathrm{cm}$ coincide com o ponto de apoio dos pés. $\mathrm{O}$ avaliado retirava o calçado e na posição sentada tocava os pés na caixa com os joelhos estendidos. Com ombros flexionados, cotovelos estendidos e mãos sobrepostas executava a flexão do tronco à frente devendo este tocar o ponto máximo da escala com as mãos. Foram realizadas três tentativas sendo considerada apenas a melhor marca. Os sujeitos foram submetidos a uma única sessão de avaliação.

Os testes foram realizados entre 2005 e 2007 por 8 avaliadores devidamente treinados, com pelo menos 6 meses de experiência nos procedimentos da coleta que participaram de todas as medidas durante todo o período de coleta.

\section{Grupos}

Além da idade e gênero, os grupos foram divididos também pelo nível de atividade física, pois segundo Rubini et al. ${ }^{10}$ são estes os fatores que mais influenciam na flexibilidade.

O grupo sedentário foi determinado por aqueles que não praticavam atividade física supervisionada enquanto que o grupo ativo foi composto daqueles que praticavam atividade física supervisionada de 1 a 7 vezes por semana. Embora o nível de atividade física deva ser determinado além da freqüência semanal pela intensidade e duração da atividade ${ }^{11-13}$ este fato não foi considerado neste estudo, pois os achados de Bandy et al. ${ }^{14}$, Okada et al. ${ }^{15}$ e Melo et al..$^{16}$ sugerem não haver diferença nos níveis de flexibilidade de sujeitos ativos e muito ativos, mas somente entre sedentários e ativos. Além disso, ainda não está claro na literatura quais os tipos, freqüências, intensidades e durações ideais do treinamento exclusivo de flexibilidade para melhora da saúde ou qualidade de vida.

Esta divisão ocorreu apenas para garantir que a amostra, quanto ao nível de atividade física, fosse heterogênea, uma vez que comprovadas as diferenças entre sedentários e ativos, a opção por apenas um destes níveis talvez não refletisse a população estudada, podendo influenciar os resultados.

\section{Pontos de corte}

Para a classificação dos indivíduos quanto ao nível de flexibilidade apresentado no teste utilizou-se a tabela proposta pelo CSTF.

A partir destes dados foi elaborada uma nova tabela de classificação para TSA seguindo os mesmos critérios estabelecidos pela classificação canadense, i.e., calculou-se os percentis divididos em 5 categorias: Fraco (até $20^{\circ}$ percentil), Abaixo da Média $\left(25^{\circ}\right.$ ao $\left.40^{\circ}\right)$, Média $\left(45^{\circ}\right.$ ao $\left.60^{\circ}\right)$, Acima da Média $\left(65^{\circ}\right.$ ao $\left.80^{\circ}\right)$ e Excelente (acima de $85^{\circ}$ ).

\section{RESULTADOS}

Os dados referentes à amostra são apresentados na tabela 1. No grupo feminino, a faixa etária de 40 a 49 anos em diante foi na sua maioria ativa. Já no masculino, em todas as faixas etárias a quantidade de ativos foi superior a de sedentários.

A tabela 2 apresenta os resultados do TSA de acordo com o gênero, idade e nível de atividade física.

A tabela 3 classifica os resultados usando o que propõe o CSTF. Observou-se que tanto para o masculino quanto para o feminino, nenhuma faixa etária apresentou índice de flexibilidade correspondente à média proposta pela classificação canadense e a faixa etária que corresponde aos 70 anos ou mais não foi classificada, pois o CSTF não apresenta índices para esta faixa.

Estes resultados foram distribuídos em percentis que se encontram na Tabela 4, estabelecendo-se pontos de corte nos percentuais 20, 40,60, 80 e 100. Com base nestes dados, foi confeccionada a tabela normativa do nível de flexibilidade para o TSA proposta por este estudo (Tabela 5).

Tabela 1. Distribuição dos indivíduos quanto ao nível de atividade física de acordo com gênero e idade.

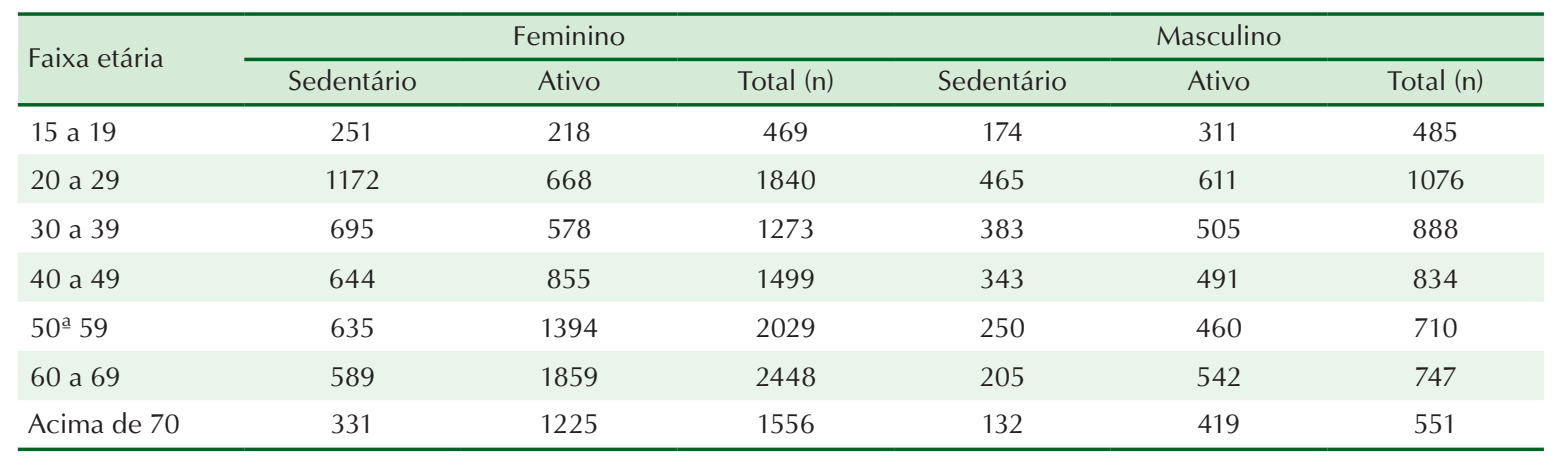


Tabela 2. Valores médios ( \pm DP) de flexibilidade $(\mathrm{cm})$ para o teste de sentar e alcançar de homens e mulheres em diferentes faixas etárias e níveis de atividade física.

\begin{tabular}{|c|c|c|c|c|c|c|c|c|}
\hline \multirow{2}{*}{$\frac{\text { Faixa etária }}{15 \text { a } 19}$} & \multicolumn{4}{|c|}{ Feminino } & \multicolumn{4}{|c|}{ Masculino } \\
\hline & $24,11 \pm$ & 9,89 & $28,45 \pm$ & 9,84 & $21,68 \pm$ & 9,88 & $23,50 \pm$ & 9,49 \\
\hline 20 a 29 & $23,55 \pm$ & 9,43 & $27,01 \pm$ & 9,84 & $19,62 \pm$ & 9,73 & $22,90 \pm$ & 9,72 \\
\hline 30 a 39 & $25,02 \pm$ & 9,46 & $26,86 \pm$ & 9,13 & $20,59 \pm$ & 9,92 & $23,15 \pm$ & 9,98 \\
\hline 50 a 59 & $23,13 \pm$ & 9,30 & $25,84 \pm$ & 9,11 & $15,47 \pm$ & 9,30 & $18,56 \pm$ & 10,10 \\
\hline 60 a 69 & $21,07 \pm$ & 9,88 & $23,31 \pm$ & 9,35 & $13,73 \pm$ & 9,49 & $17,41 \pm$ & 10,25 \\
\hline 70 ou mais & $17,14 \pm$ & 9,53 & $22,06 \pm$ & 8,69 & $11,74 \pm$ & 9,37 & $13,10 \pm$ & 9,63 \\
\hline
\end{tabular}

Tabela 3. Classificação do nível de flexibilidade em $\mathrm{cm}$ para o teste de sentar e alcançar de homens e mulheres em diferentes faixas etárias de acordo com o Canadian Standardizes Test of Fitness (CSTF).

\begin{tabular}{ccccc}
\hline \multirow{2}{*}{ Faixa etária } & \multicolumn{2}{c}{ Feminino } & & \multicolumn{2}{c}{ Masculino } \\
\cline { 2 - 5 } & Flexibilidade $(\mathrm{cm})$ & CSTF & Flexibilidade & CSTF \\
\hline 15 a 19 & $26,130 \pm 10,111$ & Fraco & $22,848 \pm 9,648$ & Fraco \\
20 a 29 & $24,805 \pm 9,684$ & Fraco & $21,480 \pm 9,905$ & Fraco \\
30 a 39 & $25,855 \pm 9,716$ & Fraco & $22,046 \pm 9,703$ & Fraco \\
40 a 49 & $25,396 \pm 9,547$ & Abaixo da média & $19,935 \pm 9,192$ & Abaixo da média \\
50 a 59 & $24,990 \pm 9,379$ & Abaixo da média & $17,474 \pm 9,868$ & Abaixo da média \\
60 a 69 & $22,768 \pm 9,627$ & Abaixo da média & $16,396 \pm 10,136$ & Abaixo da média \\
Acima de 70 & $21,012 \pm 9,611$ & Não classificado & $12,775 \pm 9,425$ & Não classificado \\
\hline
\end{tabular}

CSTF $=$ Canadian Standardizes Test of Fitness.

Tabela 4. Valores da flexibilidade $(\mathrm{cm})$ para o teste de sentar e alcançar distribuídos em percentis conforme idade e gênero.

\begin{tabular}{|c|c|c|c|c|c|c|c|c|c|c|c|c|c|c|}
\hline $\begin{array}{l}\text { Idade } \\
\text { Gênero }\end{array}$ & \multicolumn{2}{|c|}{$15-19$} & \multicolumn{2}{|c|}{$20-29$} & \multicolumn{2}{|c|}{$30-39$} & \multicolumn{2}{|c|}{$40-49$} & \multicolumn{2}{|c|}{$50-59$} & \multicolumn{2}{|c|}{$60-69$} & \multicolumn{2}{|c|}{$>70$} \\
\hline 95 & 42 & 39 & 41 & 37 & 41 & 38 & 41 & 36 & 40 & 35 & 38 & 35 & 36 & 30 \\
\hline 90 & 40 & 36 & 37 & 35 & 38 & 34 & 37 & 32 & 37 & 32 & 35 & 31 & 33 & 26 \\
\hline 85 & 37 & 33 & 35 & 32 & 36 & 32 & 36 & 30 & 35 & 28 & 33 & 28 & 31 & 23 \\
\hline 75 & 34 & 29 & 32 & 28 & 33 & 29 & 32 & 27 & 32 & 24 & 30 & 23 & 28 & 19 \\
\hline 70 & 32 & 28 & 30 & 27 & 32 & 28 & 31 & 25 & 31 & 22 & 28 & 21 & 27 & 18 \\
\hline 65 & 30 & 27 & 29 & 26 & 30 & 26 & 30 & 24 & 29 & 21 & 27 & 20 & 25 & 16 \\
\hline 60 & 29 & 25 & 27 & 24 & 29 & 25 & 28 & 22 & 28 & 20 & 26 & 18 & 24 & 15 \\
\hline 45 & 25 & 21 & 24 & 20 & 25 & 21 & 25 & 18 & 24 & 16 & 22 & 14 & 20 & 10 \\
\hline 40 & 24 & 20 & 23 & 19 & 24 & 19 & 23 & 17 & 23 & 14 & 21 & 13 & 19 & 9 \\
\hline 35 & 23 & 19 & 21 & 18 & 22 & 18 & 22 & 16 & 22 & 13 & 19 & 11 & 17 & 8 \\
\hline 30 & 21 & 17 & 20 & 16 & 21 & 17 & 21 & 15 & 21 & 12 & 18 & 10 & 16 & 6 \\
\hline 25 & 19 & 16 & 18 & 15 & 19 & 15 & 19 & 13 & 19 & 10 & 16 & 9 & 15 & 5 \\
\hline 20 & 18 & 14 & 17 & 13 & 17 & 13 & 17 & 12 & 17 & 9 & 15 & 7 & 13 & 3 \\
\hline 15 & 15 & 13 & 15 & 11 & 15 & 12 & 15 & 10 & 15 & 7 & 13 & 5 & 11 & 2 \\
\hline
\end{tabular}

F: feminino; M: masculino. 
Tabela 5. Valores normativos de flexibilidade $(\mathrm{cm})$ para o teste de sentar e alcançar sugeridos com base nos dados do presente estudo.

\begin{tabular}{|c|c|c|c|c|c|c|c|c|c|c|c|c|c|c|c|c|c|c|c|c|c|c|}
\hline & \multirow[b]{2}{*}{ Classificação } & \multicolumn{21}{|c|}{ Faixas etárias } \\
\hline & & \multicolumn{3}{|c|}{15 a 19} & \multicolumn{3}{|c|}{20 a 29} & \multicolumn{3}{|c|}{30 a 39} & \multicolumn{3}{|c|}{40 a 49} & \multicolumn{3}{|c|}{50 a 59} & \multicolumn{3}{|c|}{60 a 69} & \multicolumn{3}{|c|}{$\geq 70$} \\
\hline \multirow{3}{*}{ M } & Excelente & \multicolumn{3}{|c|}{$\geq 33$} & \multicolumn{3}{|c|}{$\geq 32$} & \multicolumn{3}{|c|}{$\geq 32$} & \multicolumn{3}{|c|}{$\geq 30$} & \multicolumn{3}{|c|}{$\geq 28$} & \multicolumn{3}{|c|}{$\geq 28$} & \multicolumn{3}{|c|}{$\geq 23$} \\
\hline & Média & 21 & - & 26 & 20 & - & 25 & 21 & - & 25 & 18 & - & 23 & 16 & - & 20 & 14 & - & 19 & 10 & - & 15 \\
\hline & Abaixo da média & 16 & - & 20 & 15 & - & 19 & 15 & - & 20 & 13 & - & 17 & 10 & - & 15 & 9 & - & 13 & 5 & - & 9 \\
\hline \multirow{4}{*}{$\mathrm{F}$} & Excelente & \multicolumn{3}{|c|}{$\geq 37$} & \multicolumn{3}{|c|}{$\geq 35$} & \multicolumn{3}{|c|}{$\geq 36$} & \multicolumn{3}{|c|}{$\geq 36$} & \multicolumn{3}{|c|}{$\geq 35$} & \multicolumn{3}{|c|}{$\geq 33$} & \multicolumn{3}{|c|}{$\geq 31$} \\
\hline & Acima da média & 30 & - & 36 & 29 & - & 34 & 30 & - & 35 & 30 & - & 35 & 29 & - & 34 & 27 & - & 32 & 25 & - & 30 \\
\hline & Média & 25 & - & 29 & 24 & - & 28 & 25 & - & 29 & 24 & - & 29 & 24 & - & 28 & 22 & - & 26 & 20 & - & 24 \\
\hline & Abaixo da média & 19 & - & 24 & 18 & - & 23 & 19 & - & 24 & 19 & - & 23 & 19 & - & 23 & 16 & - & 21 & 15 & - & 19 \\
\hline
\end{tabular}

$M=$ masculino; $F=$ feminino

\section{DISCUSSÃO}

Este estudo teve por objetivo identificar o nível de flexibilidade de homens e mulheres de diferentes faixas etárias através do TSA seguindo protocolo do CSTF. O principal achado foi verificar que a amostra investigada apresentou valores inferiores aos recomendados pela referida tabela em todas as faixas etárias independente do gênero. Este achado nos permite especular que brasileiros possuem menor nível de flexibilidade do que canadenses ou que a tabela geralmente utilizada na maioria dos estudos é inadequada para nossa população nos dias atuais.

Os resultados do presente estudo corroboram vários outros realizados na população brasileira que ao utilizarem o TSA de acordo com o protocolo canadense retratam situações de flexibilidade precária.

Silva e Juvêncio ${ }^{17}$, por exemplo, ao avaliarem a flexibilidade de 28 homens ( $38,9 \pm 11,1$ anos) e 18 mulheres (40,9 $\pm 9,5$ anos) obtiveram resultados considerados ruins ou abaixo da média em $72,22 \%$ de indivíduos do gênero feminino e $60,72 \%$ do masculino, sendo que os indivíduos apresentavam forte propensão ao sedentarismo.

Em outro estudo que avaliou a flexibilidade de mulheres idosas praticantes de ginástica e hidroginástica, observou-se que as praticantes de ginástica obtiveram índices de flexibilidade considerados na média, porém as que praticavam hidroginástica se encontraram abaixo da média, o que pode ser explicado pela maior freqüência e volume de trabalho com exercícios de alongamento e flexibilidade ao final das aulas de ginástica ${ }^{18}$.

Em estudo realizado por Mendonça et al. ${ }^{19} 21$ homens entre 60 e 72 anos e 23 mulheres entre 63 e 73 anos apresentaram níveis de flexibilidade considerados na média para o gênero masculino, porém o feminino apresentou classificação fraco.

Já Dias et al..$^{20}$, ao analisarem a flexibilidade de homens ( $n=132)$ e mulheres ( $n=194)$ sedentários também encontraram dados semelhantes: para o gênero masculino e feminino, nas faixas etárias de 20-29 e 30-39 a classificação foi fraca e na faixa etária de 40 a 49 foi abaixo da média.

O estudo realizado no Canadá em 1981 que serviu de base para a elaboração do manual do Canadian Physical Test classificou os participantes quanto à prática de atividade física ${ }^{21}$. Os indivíduos foram divididos em sedentários, minimamente ativos e adequadamente ativos de acordo com a média diária, nos últimos 12 meses do gasto calórico por quilograma de peso corporal. Observou-se que para todas as faixas etárias em ambos os gêneros, a quantidade de indivíduos classificados como sedentários foi superior, ficando entre 50,4\% e 69,2\%.

Já no presente estudo, para o grupo feminino o número de pessoas ativas foi maior em relação à idade. Nas categorias de 15 a 19, 20 a 29 e 30 a 39 , a quantidade de sedentários foi $53,5 \%$ a $63,6 \%$ contra $21,2 \%$ a $42,9 \%$ nas categorias de 40 a 49,50 a 59, 60 a 69 e acima de 70 anos. No masculino, o número de ativos foi superior em todas as categorias, variando de $56,8 \%$ a $76,1 \%$.

Acreditava-se que por serem predominantemente ativos, os indivíduos avaliados neste estudo apresentariam melhores índices de flexibilidade. No entanto, apesar de apresentarem índices mais elevados de flexibilidade quando comparados aos sedentários, os indivíduos ativos não chegaram a atingir a média proposta pela classificação canadense. Isso, no entanto, não foi identificado por Guedes et $\mathrm{a}^{122}$ ao avaliarem adolescentes ativos e sedentários onde a média da flexibilidade não apre- 
sentou diferença significante entre os dois grupos, concluindo que a prática habitual de atividade física nem sempre garante uma boa aptidão física. Talvez a melhora desta capacidade motora só ocorra com treino específico e não generalizado.

Assim, com mais esta evidência da inadequação da classificação proposta pelo estudo canadense, a nova tabela normativa para população paulista feita no presente trabalho preenche uma lacuna importante na literatura, embora ainda sejam necessários estudos em outras regiões que tenham a mesma finalidade.

Algumas limitações do presente trabalho devem ser observadas. Talvez o acesso de indicadores econômicos, sociais, e dos níveis da aptidão física relacionada à saúde nos permite correlacionar algumas variáveis e possivelmente fazer maiores extrapolações. Além disso, os dados devem ser cautelosamente interpretados e somente para a população investigada.

\section{CONCLUSÃO}

Apesar de os indivíduos serem em sua maioria praticantes de atividade física regular, concluímos que quando classificados de acordo com a tabela canadense de flexibilidade, os índices de flexibilidade foram precários, independente da idade e gênero. Isso demonstrou que a utilização da tabela proposta pelo CSTF de 1985 para classificação da flexibilidade em outras populações pode ser limitada. Há necessidade da construção de tabelas normativas para outros grupos populacionais como a realizada no presente trabalho que auxiliem a avaliação e prescrição do treinamento desta capacidade motora condicionante.

\section{REFERÊNCIAS}

1. Tritschler KA. Barrow \& McGee's practical measurement and assessment. 5rd edn Baltimore: Lippincott Williams \& Wilkins, 2000.

2. Haskell WL, Lee IM, Pate RR, Powell KE, Blair SN, Franklin BA, et al. Physical Activity and Public HBealth. Updated Recommendation for Adults From the American College of Sports Medicine and the American Heart Association. Circulation 2007;116(9):1081-93.

3. Nelson ME, Rejeski WJ, Blair SN, Duncan PW, Judge JO, King AC, et al. Physical Activity and Public Health in Older Adults. Recommendation From the American College of Sports Medicine and the American Heart Association. Circulation 2007;116(9):1094-105.

4. American College of Sport Medicine, Franklin BA, Whaley MH , Howley ET , Balady GJ. ACSM's guidelines for exercise testing and prescription (6th ed.). Philadelphia: Lippincott Williams \& Wilkins; 2000.
5. Sainz de Baranda P, Ayala F. Chronic flexibility improvement after 12 week of stretching program utilizing the ACSM recommendations: hamstring flexibility. Int J Sports Med 2010:31(6):389-96.

6. Wells KF, Dillon EK. The sit and reach - a test of back and leg flexibity. Res Quart. 1952;23:115-8.

7. American College of sports Medicine. ACSM`s guidelines to exercise testing and prescription 5th edn. Baltimore: Williams and Wilkins; 1995.

8. Beighton P, Solomon L, Soskolne CL. Articular mobility in an African population. Ann Rheum Dis 1973;32(5):413-8.

9. Canadian Standardized Test of Fitness (CSTF) Operations manual, 3rd edn, Fitness and Amateur Sport, Ottawa: Minister of State; 1986.

10. Rubini EC, Costa AL, Gomes OS. The effects of stretching on strength performance. Sports Med 2007;37(3):213-24.

11. Pollock ML, Gaesser GA, Butcher JD, Després JP, Dishman RK, Franklin BA, et al. American College of Sports Medicine Position Stand. The recommended quantity and quality of exercise for developing and maintaining cardiorespiratory and muscular fitness, and flexibility in healthy adults. Med Sci Sports Exerc 1998; 30(6):975-91.

12. Jakicic JM, Clark K, Coleman E, Donnelly JE, Forevt J, Melanson E, et al. American College of Sports Medicine position stand. Appropriate intervention strategies for weight loss and prevention of weight regain for adults. Med Sci Sports Exerc. 2001;33(12):2145-56.

13. Donnelly JE, Blair SN, Jakicic JM, Manore MM, Rankin JW, Smith BK. Appropriate Physical Activity Intervention Strategies for Weight Loss and Prevention of Weight Regain for Adults. Med Sci Sports Exerc 2009;41(2):459-71.

14. Bandy WD, Irion JM, Briggler M. The Effect of Time and Frequency of Static Stretching on Flexibility of the Hamstring Muscles. Phys Ther1997;77(10):1090-96.

15. Okada GT, Aquino Junior AE, Barreto SMG, Duarte ACGO, Silva RG. Resultado de diferentes freqüências semanais de treinamento sobre a composição corporal e aptidão física em mulheres pré-menopausais entre 30 e 50 anos. Motriz 2008;14(3):241-51.

16. Melo FAP, Oliveira FMF, Almeida MB. Nível de atividade física não identifica o nível de flexibilidade de adolescentes. Rev Bras Ativ Fis Saúde 2009;14(1):48-54.

17. Silva CD, Juvêncio, JF. Diagnóstico da aptidão física relacionada à saúde em trabalhadores de escritório da Universidade Federal de Viçosa. Rev Bras Cineantropom Desempenho Hum 2004;6(1):63-71.

18. Almeida APPV, Veras RP, Doimo LP. Avaliação do equilíbrio estático e dinâmico de idosas praticantes de hidroginástica e ginástica. Rev Bras Cineantropom Desempenho Hum 2010;12(1):55-61.

19. Mendonça TT, Ito RE, Bartholomeu T, Tinucci T, Forjaz C. Risco cardiovascular, aptidão física e prática de atividade física de idosos de um parque de São Paulo. Rev Bras Ciên Mov 2004;12(2):19-24. 
20. Dias DF, Reis ICB, Reis DA, Cyrino ES, Ohara D, Carvalho FO, Casonatto J, Loch MR. Comparação da aptidão física relacionada à saúde de adultos de diferentes faixas etárias. Rev Bras Cineantropom Desempenho Hum 2008;;10(2):123-8.

21. Stephens T, Craig CL, Ferris BF. Adult physical activity in Canada: findings from the Canada Fitness Survey I. Can J Public Health1986;77(4):285-90.

22. Guedes DP, Guedes JERP, Barbosa DS, Oliveira JA. Atividade física habitual e aptidão física relacionada à saúde em adolescentes. Rev Bras Ciên Mov 2002;10(1):13-21.
Endereço para correspondência

Cibele Calvi Anic Ribeiro

Rua Euclides Pacheco, 2034. Tatuapé CEP: 03321-001. São Paulo, SP. Brasil.

E-mail: cibeleanic@hotmail.com 